\title{
On the Poisson distribution.
}

\author{
By Hisao Watanabe
}

(Receivd Nov. 25, 1955)

Let $\cdots, x_{-1}, x_{0}, x_{1}, \cdots$ be the points on the real line such that $\cdots x_{-1}<x_{0}<x_{1} \cdots\left(x_{0} \equiv 0\right)$. Then if $\left\{x_{j}-x_{j-1}\right\} \quad(j=0,1,2, \cdots)$ are independent random variables with common distribution function $F(x)$, where $F(x)$ is the distribution function of a non-negative random variable with $F(-0)=0, F(\infty)=1$, we shall say that these points are distributed at random according to $F(x)$.

Now consider a system of particles $P_{n}(n=0, \pm 1, \pm 2, \cdots)$ which start from the above stated random positions $x_{n}(n=0, \pm 1, \pm 2, \cdots)$. When we denote by $X_{n}(t)$ the displacement of the $n$-th particle $P_{n}$ up to the time $t$, the coordinate $Y_{n}(t)$ of the particle at the time $t$ is

$$
Y_{n}(t)=x_{n}+X_{n}(t), \quad X_{n}(0)=0, \quad t \geq 0 .
$$

In the following, let us confine ourselves to the discrete time parameter $t=0,1,2, \cdots$, and we shall impose the following conditions on the movement of the particles. The random variables $X_{n}(t)-X_{n}(t-1)$ are mutually independent for each $n, t,-\infty<n<\infty, t \geqq 0$, and obey the same distribution function $G(x)$ for all $n, t$, moreover, for each $t>0$ the classes of random variables

$$
\left\{X_{n}(t), n=0, \pm 1, \pm 2, \cdots\right\} \quad\left\{x_{n}, n=0, \pm 1, \pm 2, \cdots\right\}
$$

are mutually independent.

By the Fourier analytical method [2], Prof. Maruyama [3] investigated the limiting distribution of the number $N_{I}(t)$ of particles lying in an interval $I=[a, b]$ at $t$ under the condition that $G(x)$ is a non-lattice distribution function. In this note, we shall discuss the problem when $G(x)$ is a lattice distribution function with maximum span $d>0$.

THEOREM. If $0<m=\int_{-\infty}^{+\infty} x d F(x)<\infty$, then we have 


$$
\lim _{t \rightarrow \infty} \operatorname{Pr}\left\{N_{I}(t)=k\right\}=e^{-\lambda} \frac{\lambda^{k}}{k !} \quad k=0,1,2, \cdots,
$$

with

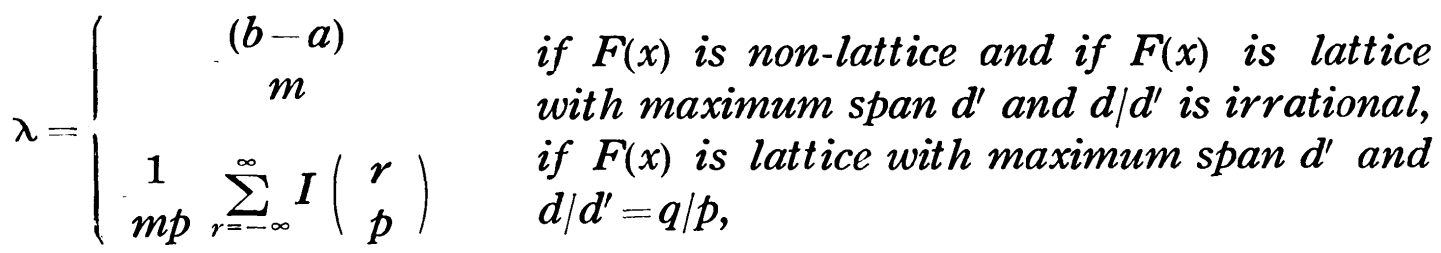

where

$$
I(x)= \begin{cases}1 & x \in[a, b] \\ 0 & x \in[a, b] .\end{cases}
$$

Proof. Our discussion proceeds analogously to [3]. By (1), we can write

$$
N_{l}(t)=\sum_{n=-\infty}^{\infty} I\left(Y_{n}(t)\right)
$$

As in [2], we introduce a non-negative smooth function $H(x)$ satisfying the following conditions (i), (ii).

(i) Fourier transformations

$$
\begin{aligned}
& \int_{-\infty}^{\infty} H(x) e^{-i t x} d x=h(t), \\
& \frac{1}{2 \pi} \int_{-\infty}^{\infty} h(t) e^{i t x} d x=H(x)
\end{aligned}
$$

are absolutely convergent and the equalities hold for all $t$ and $x$. (ii) $h(t)$ vanishes outside a finite interval $[-c, c]$.

Let us put

$$
N_{H}(t)=\sum_{n=-\infty}^{\infty} H\left(Y_{n}(t)\right) \text {. }
$$

We shall begin with proving the existence of $E\left\{N_{I}(t)\right\}$ for all $t \geqq 0$ and $\lim _{t \rightarrow \infty} E\left\{N_{I}(t)\right\}$. If we denote the characteristic functions of $F(x)$ and $G(x)$ by $\varphi(u)$ and $\psi(u)$ respectively, we have

$$
E\left\{H\left(Y_{n}(t)\right\}=E\left\{\frac{1}{2 \pi} \int_{-\infty}^{\infty} e^{i u\left(x_{n}+X_{n}^{(t)}\right)} h(u) d u\right\}\right.
$$


(3)

$$
= \begin{cases}\frac{1}{2 \pi} \int_{-\infty}^{\infty} \Phi^{n}(u) \psi^{t}(u) h(u) d u & n \geqq 0 \\ \frac{1}{2 \pi} \int_{-\infty}^{\infty} \Phi^{n}(-u) \psi^{t}(u) h(u) d u & n \leqq 0 .\end{cases}
$$

If we write for $0<\rho<1$,

$$
N_{H, \rho}(t)=\sum_{n=-\infty}^{\infty} \rho^{|n|} H\left(Y_{n}(t)\right),
$$

then

$$
\lim _{\rho \rightarrow 1} N_{H, \rho}(t)=N_{H}(t),
$$

and in view of (3) we get

$$
E\left\{N_{H, \rho}(t)\right\}=I(\rho, t)+J(\rho, t)+K(t),
$$

where

$$
\begin{aligned}
& I(\rho, t)=\frac{1}{\pi} \int_{-e}^{c} \frac{1-\rho}{Q(\rho, u)} h(u) \psi^{t}(u) d u, \\
& J(\rho, t)=\frac{\rho}{\pi} \int_{-c}^{e} \frac{1-a(u)}{Q(\rho, u)} h(u) \psi^{t}(u) d u, \\
& K(t)=-\frac{1}{2 \pi} \int_{-e}^{c} h(u) \psi^{t}(u) d u, \\
& a(u)=\int_{0}^{\infty} \cos u x d F(x) \text { and } Q(\rho, u)=|1-\rho \varphi(u)|^{2} .
\end{aligned}
$$

First suppose that $F(x)$ is a non-lattice distribution function. Then, by the same analysis as in [3] p. 3, we have

$$
\lim _{\rho \rightarrow 1-0} I(\rho, t)=\frac{h(0)}{m}
$$

and also

$$
\lim _{\rho \rightarrow 1-0} J(\rho, t)=\frac{1}{\pi} \int_{-c}^{c} \frac{1-a(u)}{Q(1, u)} h(u) \psi^{t}(u) d u .
$$

Since $|\psi(u)|<1$ except for $u=2 \nu \pi / d(\nu=0, \pm 1, \pm 2, \cdots)$, we have by the convergence theorem of Lebesgue

$$
\lim _{t \rightarrow \infty} \lim _{\rho \rightarrow 1-0} J(\rho, t)=0, \quad \lim _{t \rightarrow \infty} K(t)=0 \text {. }
$$


Combining these we obtain

$\lim _{t \rightarrow \infty} \lim _{\rho \rightarrow 1-0} E\left\{N_{\rho, H}(t)\right\}=\lim _{t \rightarrow \infty} E\left\{N_{H}(t)\right\}=\begin{gathered}h(0) \\ m\end{gathered}=\frac{1}{m} \int_{-\infty}^{\infty} H(x) d x$.

Next suppose that $F(x)$ is a lattice distribution with maximum span $d^{\prime}$, in which case the situation is different. We may suppose without loss of generality that $d^{\prime} \equiv \mathbf{1}$ by changing the scale. Then, $I(\rho, t)$ can be written as

$$
\begin{aligned}
I(\rho, t) & =\frac{1}{\pi} \sum_{\nu=-\infty}^{\infty} \int_{(2 \nu-1) \pi}^{(2 \nu+1) \pi} h(u) \psi^{t}(u) \frac{1-\rho}{Q(\rho, u)} d u \\
& =\frac{1}{\pi} \int_{-\pi}^{\pi} \sum_{\nu=-\infty}^{\infty} h(u+2 \nu \pi) \psi^{t}(u+2 \nu \pi) \frac{1-\rho}{Q(\rho, u)} d u .
\end{aligned}
$$

Since $S(u)=\sum_{\nu=-\infty}^{\infty} h(u+2 \nu \pi) \psi^{t}(u+2 \pi)$ is continuous with respect to $u$, the analysis in [3] p. 5 gives

$$
\lim _{\rho \rightarrow 1-0} I(\rho, t)=\frac{S(0)}{m}=\frac{1}{m} \sum_{\nu=-\infty}^{\infty} h(2 \nu \pi) \psi^{t}(2 \nu \pi) .
$$

Firstly, let $d$ be irrational. Then, since $j / d$ cannot be an integer for any $j(j= \pm 1, \pm 2, \cdots)$, we have $|\psi(2 \nu \pi)|<1$ for any $\nu(\nu= \pm 1, \pm 2, \cdots)$ and

$$
\lim _{t \rightarrow \infty} \lim _{\rho \rightarrow 1-0} I(\rho, t)=\lim _{t \rightarrow \infty} \frac{1}{m} \sum_{\nu=-\infty}^{\infty} h(2 \nu \pi) \psi^{t}(2 \nu \pi)=\frac{h(0)}{m} .
$$

On the other hand, when $d$ is rational, we can write $d=q / p$ with relatively prime positive integers $p, q$. But $j / d$ can be an integer when and only when $j=k q(k=0, \pm 1, \pm 2, \cdots)$, and $\psi(u)$ has $2 \pi / d$ as a period. Hence

$$
\begin{aligned}
\lim _{t \rightarrow \infty} & \lim _{\rho \rightarrow 1-0} I(\rho, t)=\lim _{t \rightarrow \infty} \frac{1}{m} \sum_{\nu=-\infty}^{\infty} h(2 \nu \pi) \psi^{t}(2 \nu \pi) \\
& =\frac{1}{m} \sum_{k=-\infty}^{\infty} h\left(2 k q \frac{\pi}{d}\right)=\frac{1}{m} \sum_{k=-\infty}^{\infty} h(2 \pi k p) .
\end{aligned}
$$

Poisson's summation formula [1]

1) Since $h(u)$ vanishes outside $(-c, c), \Sigma$ is essentially a summation over a finite number of $\nu$. 


$$
\sum_{k=-\infty}^{\infty} h(2 \pi k p)=\sum_{r=-\infty}^{\infty} \frac{r}{p} H\left(\frac{r}{p}\right)
$$

applied to the last term gives us

$$
\lim _{t \rightarrow \infty} \lim _{\rho \rightarrow 1-0} I(\rho, t)=\frac{1}{m} \sum_{r=-\infty}^{\infty} \frac{1}{p} H\left(\frac{r}{p}\right) .
$$

Next we observe that

$$
\lim _{\rho \rightarrow 1-0} J(\rho, t)=\frac{1}{\pi} \int_{-\pi}^{\pi} \sum_{r=\infty}^{\infty} h(u+2 \nu \pi) \psi^{t}(u+2 \nu \pi) \frac{1-a(u)}{Q(1, u)} d u .
$$

Then, since $\lim _{t \rightarrow \infty} \sum_{\nu=-\infty}^{\infty} h(u+2 \nu \pi) \psi^{t}(u+2 \nu \pi)=0$ except at most for a finite number of $u$ in $(-\pi, \pi)$ and $1-a(u) / Q(1, u)$ is integrable in that interval, we get

$$
\lim _{t \rightarrow \infty} \lim _{\rho \rightarrow 1-0} J(\rho, t)=0,
$$

and also

$$
\lim _{t \rightarrow \infty} K(t)=\lim _{t \rightarrow \infty} \frac{-\mathbf{1}}{\pi} \int_{-c}^{c} h(u) \psi^{t}(u) d u=0 .
$$

Thus, we have established:

(A) If $F(x)$ is a non lattice distribution,

$$
\lim _{t \rightarrow \infty} E\left\{N_{H}(t)\right\}=\frac{1}{m} \int_{-\infty}^{\infty} H(x) d x .
$$

(B) If $F(x)$ is a lattice distribution,

$$
\lim _{t \rightarrow \infty} E\left\{N_{H}(t)\right\}= \begin{cases}\frac{1}{m} \int_{-\infty}^{\infty} H(x) d x & \text { if } d \text { is irrational } \\ \frac{1}{m p} \sum_{r=-\infty}^{\infty} H\left(\frac{r}{p}\right) & \text { if } d=q / p .\end{cases}
$$

In the case of $(\mathbf{A})$ and $(\mathbf{B})$ with irrational $d$, we obtain by the argument in [3]

$$
\lim _{t \rightarrow \infty} E\left\{\exp \left(i z N_{I}(t)\right)\right\}=\exp \left\{\frac{(b-a)}{m}\left(e^{i z}-1\right)\right\},
$$

and therefore 


$$
\begin{gathered}
\lim _{t \rightarrow \infty} \operatorname{Pr}\left\{N_{l}(t)=k\right\}=e^{-\lambda} \cdot \frac{\lambda^{k}}{k !} \quad(k=0,1,2, \cdots) \\
\lambda=\frac{(b-a)}{m} .
\end{gathered}
$$

In the remaining case of $(\mathbf{B})$, we proceed as in [2], with modifications of the approximation procedures used there. We define a continuous non-negative function $H_{0}(x)$ which vanishes outside a finite interval $(-k, k)$ and satisfies

$$
0<H_{0}(x)-I(x) \quad \text { for } \quad x \in I
$$

and for sufficiently small $\eta>0$

$$
\sum_{r=-\infty}^{\infty}\left(H_{0}\left(\begin{array}{c}
r \\
p
\end{array}\right)-I\left(\begin{array}{c}
r \\
p
\end{array}\right)\right)<\eta
$$

Let us write

$$
H_{0}^{*}(x)=\int_{-\infty}^{\infty} H_{0}(x-y) K_{\lambda}(y) d y, \quad K_{\lambda}=\frac{\sin ^{2} \lambda x / 2}{\pi \lambda x^{2} / 2} .
$$

Next take an interval $J \supset I$ and define

$$
\begin{array}{cc}
H_{1}(x)=0 & \text { if } \quad x \in J \\
=H_{0}^{*}(x)-I(x) & \text { if } x \in J, \\
H_{0}^{*}(x)-I(x)=H_{1}(x)+H_{2}(x), & -\infty<x<\infty .
\end{array}
$$

Then for sufficiently large $\lambda>0$ and with a constant $c$ it holds that

$$
H_{2}(x) \leqq c \int_{-\infty}^{\infty} \begin{gathered}
1 \\
\lambda
\end{gathered} K_{\lambda^{\prime}}(x-y) d y=\bar{H}_{2}(x)
$$

Further, we define a continuous function $H_{3}(x)$ such that it vanishes outside a finite interval and

$$
\begin{array}{cl}
0<H_{3}(x)-H_{1}(x) & \text { for } x \in J, \\
\sum_{-\infty}^{\infty}\left(H_{3}\left(\frac{r}{p}\right)-H_{1}\left(\begin{array}{c}
r \\
p
\end{array}\right)\right)<\eta \quad \text { for a sufficiently small } \eta>0,
\end{array}
$$

and let

$$
H_{3}^{*}(x)=\int_{-\infty}^{\infty} H_{3}(x-y) K_{\lambda}(y) d y
$$


Then, since $H_{0}^{*}(x), H_{3}^{*}(x)$ converge uniformly to $H_{0}(x), H_{3}(x)$ respectively in any finite interval as $\lambda \rightarrow \infty$, there exists $\lambda_{0}$ such that for any $\lambda>\lambda_{0}$ and $\eta>0$

$$
\begin{aligned}
& \left|\sum_{r=-\infty}^{\infty}\left(H_{0}\left(\frac{r}{p}\right)-H_{0}^{*}\left(\frac{r}{p}\right)\right)\right|<\eta, \\
& I(x) \leqq H_{0}^{*}(x), \quad-\infty<x<\infty, \\
& \left|\sum_{r=-\infty}^{\infty}\left(H_{3}\left(\frac{r}{p}\right)-H_{3}^{*}\left(\frac{r}{p}\right)\right)\right|<\eta
\end{aligned}
$$

and

$$
H_{1}(x) \leqq H_{3}^{*}(x)
$$

Now (4), (5), (6) give us

$$
\begin{aligned}
0 & <N_{I}(t) \leqq N_{H_{0}}^{*}(t), \\
0 & <N_{H_{0}}^{*}(t)-N_{I}(t)=N_{H_{0}^{*}-I}^{*}(t) \\
= & N_{H_{2}}(t)+N_{H_{1}}(t) \leqq N_{\bar{H}_{2}}(t)+N_{H_{3}^{*}}^{*}(t) .
\end{aligned}
$$

Remembering that $H_{0}^{*}(x), \bar{H}_{2}(x), H_{3}^{*}(x)$ and their Fourier transformations $h_{0}^{*}(t), \bar{h}_{2}(t)$, (h⿱⺈ $\left.h_{3}^{*} t\right)$ satisfy (i), (ii), we get

$$
\begin{aligned}
& \lim _{t \rightarrow \infty}\left(N_{\bar{H}_{2}}(t)+N_{H_{3}^{*}}^{*}(t)\right) \leqq 0\left(\lambda^{-1}\right)+\frac{4 \eta}{m p}, \\
& \lim _{t \rightarrow \infty} N_{H_{0}^{*}}(t) \leqq \frac{1}{m p} \sum_{r=-\infty}^{\infty} I\left(\frac{r}{p}\right)+\frac{2 \eta}{m p} .
\end{aligned}
$$

Hence

$$
0<\lim _{t \rightarrow \infty} N_{I}(t)-\lim _{t \rightarrow \infty} N_{I}(t)<0\left(\lambda^{-1}\right)+\frac{4 \eta}{m p} .
$$

Taking $\lambda^{-1}+\eta$ sufficiently small and taking into account of (7)-(9), we finally obtain

$$
E\left\{N_{I}(t)\right\}<\infty \quad \text { for all } t \geqq 0
$$

and

$$
\lim _{t \rightarrow \infty} E\left\{N_{I}(t)\right\}=\frac{1}{m p} \sum_{r=-\infty}^{\infty} I\left(\frac{r}{p}\right) .
$$

By using (10), in the same way as in [3], we can show that 


$$
\begin{gathered}
\lim _{t \rightarrow \infty} \operatorname{Pr}\left\{N_{l}(t)=k\right\}=e^{-\lambda} \frac{\lambda^{k}}{k !} \quad k=0,1,2, \cdots \\
\lambda=\sum_{m p}^{1} \sum_{r=-\infty}^{\infty} I\left(\begin{array}{c}
r \\
p
\end{array}\right) .
\end{gathered}
$$

I express my sincerest thanks to Professor G. Maruyama who has suggested this investigation and given valuable advices.

\section{Deffence Academy.}

\section{Reference}

[.1] S. Bochner, Vorlesungen über Fouriersche Integrale, Leipzig, 1932.

[2] G. Maruyama, Fourier analytic treatment of some prblems on the sums of random variables, Natural Sci. Rep. Ochanomizu Univer, 6 (1955).

[3] G. Maruyama, On the Poisson distribution derived from independent random walks, ibid. 6 (1955). 